\title{
Endovascular Approach in Patients with Acute Complete Occlusion Due to Middle Cerebral Artery Dissection
}

\author{
Kang-Hoon Park, ${ }^{1}$ Hyo Sung Kwak, ${ }^{2}$ Jung-Soo Park' \\ Departments of Neurosurgery and Research Institute of Clinical Medicine of Jeonbuk National University-Biomedical Research Institute of \\ Jeonbuk National University Hospital,' Jeonju, Korea \\ Department of Radiology and Research Institute of Clinical Medicine of Jeonbuk National University-Biomedical Research Institute of Jeonbuk \\ National University Hospital, ${ }^{2}$ Jeonju, Korea
}

Objective : Dissection of the middle cerebral artery (MCA) is less common than dissection of vessels in the vertebrobasilar system or carotid artery. Acute complete occlusion related to MCA dissection is extremely rare. We report an endovascular approach in patients with acute complete occlusion due to MCA dissection.

Methods : We reviewed retrospectively the endovascular procedure and clinical results for acute-stroke patients who underwent recanalization from October 2014 through December 2018. Initial imaging findings and the endovascular procedure were analyzed for patients with acute complete occlusion due to MCA dissection.

Results : We undertook first-line aspiration thrombectomy using a Penumbra catheter in 294 patients with acute occlusion of the M1 segment. Of these patients, seven were confirmed to have acute complete occlusion due to MCA dissection. All patients had angiographic findings of an intimal flap at the proximal occlusion site of the MCA. One patient complained of severe headache during microcatheter passage through the occluded lesion and died due to massive bleeding caused by rupture of the false lumen. The remaining patients underwent initial contact aspiration thrombectomy without microcatheter passage. After aspiration thrombectomy, six patients had delayed flow through the MCA. One patient underwent stenting of the MCA because of progressive symptoms.

Conclusion : An intimal flap at the proximal portion of an occluded MCA can suggest the possibility of MCA dissection. Contrast aspiration thrombectomy without microcatheter passage can reduce the risk of false lumen rupture in cases of MCA dissection.

Key Words : Stroke · Middle cerebral artery · Dissection · Thrombectomy.

\section{INTRODUCTION}

Dissection of the middle cerebral artery (MCA) is an uncommon cause of stroke, occurring less frequently than dis- section within the vertebrobasilar system or carotid artery". Patients presenting with an intracranial dissection tend to be relatively young and have symptoms such as ischemia and subarachnoid hemorrhage (SAH). Patients with dissecting an-

- Received : February 27, $2020 \cdot$ Revised : April 14, 2020 •Accepted : June 2, 2020

- Address for reprints : Hyo Sung Kwak

Radiology and Research Institute of Clinical Medicine of Jeonbuk National University-Biomedical Research Institute of Jeonbuk National University Hospital, 567 Baekje-daero, Deokjin-gu, Jeonju 54896, Korea

Tel : +82-63-250-2582, Fax : +82-63-272-0481, E-mail : kwak8140@jbnu.ac.kr, ORCID : https://orcid.org/0000-0002-7228-8117

This is an Open Access article distributed under the terms of the Creative Commons Attribution Non-Commercial License (http://creativecommons.org/licenses/by-nc/4.0) which permits unrestricted non-commercial use, distribution, and reproduction in any medium, provided the original work is properly cited. 
eurysms of the MCA present with bleeding events, such as SAH and/or intracranial hemorrhage (ICH) ${ }^{6,11)}$. An intracranial dissection without aneurysmal dilation commonly presents with symptoms of ischemia ${ }^{2,7)}$.

Historically, conventional angiography has been considered the gold standard for the diagnosis of dissections, but it is expensive and invasive ${ }^{12)}$. Although the angiographic appearance of dissections is often clear, it cannot be used to assess the vessel wall for an intramural hematoma or thrombosis. Because of this limitation, dissections in unusual locations or with atypical morphology may be misclassified or attributed to other conditions ${ }^{12)}$.

Recently, high-resolution cross-sectional magnetic resonance imaging (MRI) has emerged as a potential method for imaging of atherosclerotic plaques in the $\mathrm{MCA}^{8}$. In particular, the initial angiographic findings of patients with a dissection of the MCA related to acute stroke typically show severe stenosis with an intimal flap or double lumen. However, MCA dissection rarely presents as complete occlusion similar to acute stroke caused by cardioembolism or atherosclerosis. Therefore, diagnosing MCA dissection in cases of complete occlusion and planning the recanalization procedure can be a challenge to interventional neuroradiologists. The aims of this study were to investigate the initial angiographic findings of patients with MCA dissection presenting as complete occlusion and acute ischemic stroke and to evaluate the recanalization strategy.

\section{MATERIALS AND METHODS}

The Institutional Review Board of Jeonbuk National University Hospital approved this retrospective study (JUH 201910-041).

\section{Patients}

We reviewed retrospectively the endovascular procedure and clinical results for acute stroke patients who underwent recanalization from October 2014 through December 2018. During this period, we selected patients with acute ischemic stroke by M1 occlusion who underwent aspiration thrombectomy using a Penumbra catheter (Penumbra, Alameda, CA, USA) as first-line EVT.

\section{Definition of MCA dissection with complete oc- clusion}

We reviewed the initial and final cerebral angiographic findings of EVT. We defined MCA dissection with complete occlusion according to the following criteria : 1) a suspected intimal flap proximal to an M1 occlusion; 2) definitive double lumen and intimal flap on cerebral angiography after initial aspiration thrombectomy; and 3) exclusion of procedure-related MCA dissection after endovascular treatment (EVT).

\section{Endovascular treatment}

EVT was carried out by a single interventional neuroradiologist with 15 years of experience. Procedures were undertaken under local anesthesia through the percutaneous transfemoral route. The first patient in this study underwent advancement of a microcatheter past the M1 occlusion site for aspiration throm-

Table 1. Patient and procedural details

\begin{tabular}{lccccc}
\hline Pt. & Site & Initial NIHSS & NIHSS at discharge & mRS at 3 months & Complications or additional procedure \\
\hline 1 & $\mathrm{~L}$ & 13 & & 6 & SAH and ICH \\
\hline 2 & $\mathrm{~L}$ & 15 & 13 & 2 \\
3 & $\mathrm{R}$ & 13 & 8 & 1 \\
4 & $\mathrm{~L}$ & 5 & 2 & 3 & 0 \\
5 & $\mathrm{~L}$ & 12 & 12 & 0 & Stent \\
\hline
\end{tabular}

Pt. : patient, NIHSS : National Institutes of Health Stroke Scale, mRS : modified Rankin Scale, L : left, SAH : subarachnoid hemorrhage, ICH : intracranial hemorrhage, $R$ : right 
bectomy. Cerebral angiography after passage of the microcatheter showed extensive MCA dissection. We changed the EVT procedure after the first patient. If MCA dissection with complete occlusion was suspected following initial cerebral angiography, we advanced a Penumbra catheter to the occlusion site without passage of a microcatheter. First, we performed aspiration thrombectomy after ensuring the Penumbra catheter made contact at the occlusion site. Second, we stopped EVT if a double lumen and/or intimal flap was observed on additional angiography after aspiration thrombectomy. If no imaging findings of MCA dissection were observed, we undertook routine aspiration thrombectomy.

\section{RESULT}

Aspiration thrombectomy with a Penumbra catheter was performed in 294 patients with an M1 occlusion during the study period. Of these patients, seven (2.4\%) were confirmed to have acute complete occlusion due to MCA dissection (one patient with cerebral angiography after microcatheter passage, and six patients with cerebral angiography after initial contact aspiration thrombectomy). Patients and procedural details are outlined in Table 1.
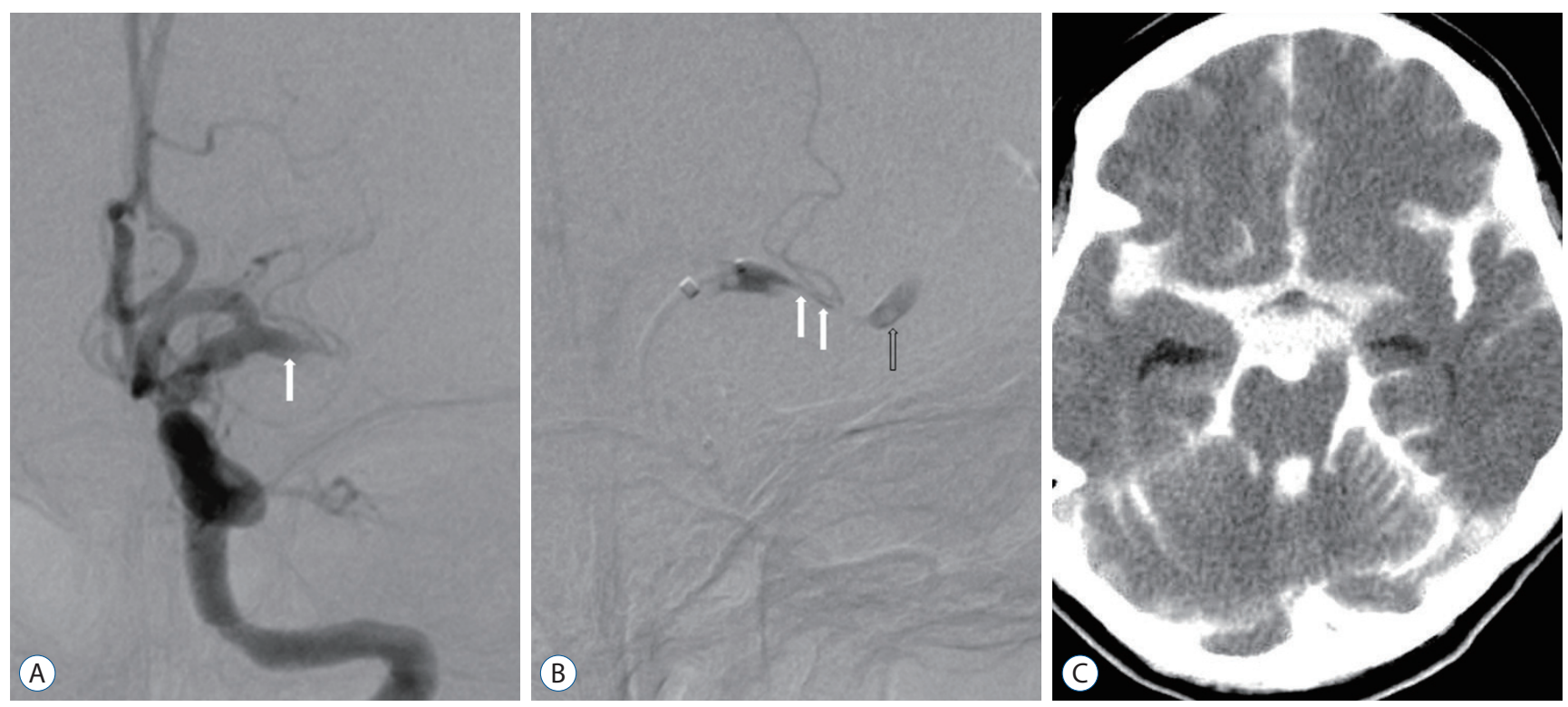

Fig. 1. A 48-year-old woman with complete occlusion of left M1. A : Left internal carotid angiogram showing complete obstruction of the left middle cerebral artery with focal intimal flap (arrow). B : Left internal carotid angiogram using microcather after patient reported severe headache during catheter passage through the obstructive lesion shows extension of the false lumen (arrows) and contrast stagnation (open arrow). C : Noncontrast computed tomography scan after interventional procedure showing massive subarachnoid hemorrhage with contrast extravasation caused by middle cerebral artery rupture.

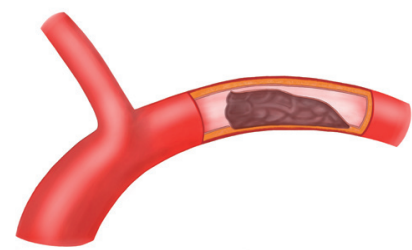

(A)

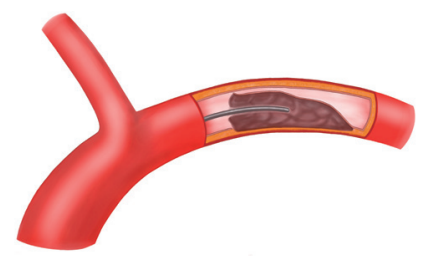

(B)

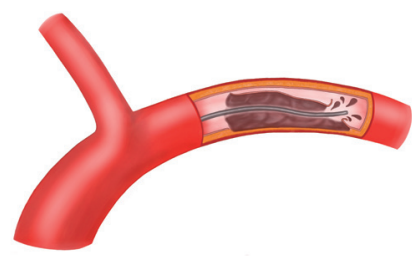

(C)

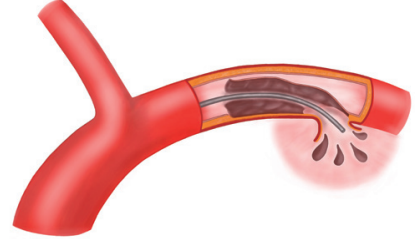

(D)

Fig. 2. A : Middle cerebral dissection. B : False lumen location of microcatheter. C : Re-entry of false lumen into the middle cerebral artery. D : Rupture of middle cerebral artery due to microcatheter in the false lumen. 


\section{Case 1}

Transfemoral cerebral angiography revealed complete obstruction of the left M1 segment (Fig. 1A) and delayed collateral flow in the distal MCA. A microcatheter was advanced for mechanical thrombectomy using the Penumbra system. The patient became irritable and complained of severe headache during passage of the catheter through the obstructive lesion. A second angiography revealed stenosis of the true lumen and a tapered pseudo-lumen, which was diagnostic for a dissection (Fig. 1B). Distal flow was delayed continuously. Follow-up angiography revealed extravasation of contrast media at the left proximal M1 portion, and the procedure was suspended. Follow-up computed tomography showed massive SAH in all cisterns (Fig. 1C). Follow-up perfusion MRI revealed diffuse infarction in the left MCA territory with increased aggravation compared to the first MRI. The patient died 6 days after cerebral angiography. We reviewed angiographic findings after her death and detected an intimal flap with complete obstruction of the true and false lumen on initial angiography. We concluded that MCA dissection with complete obstruction and massive SAH had occurred due to iatrogenic rupture of the false lumen during catheter passage through the obstructive segment. She died due to massive brain swelling (Fig. 2).

\section{Case 6}

Initial cerebral angiography revealed complete obstruction of the left M1 segment and a focal intimal flap in front of the occlusion site (Fig. 3A). Cerebral angiography after aspiration thrombectomy using a Penumbra catheter showed a double lumen and an intimal flap in the M1 segment (Fig. 3B). We did not perform an additional procedure since the entry site of the true lumen was not clear. The patient was discharged without neurologic complications.

\section{All seven patients}

All seven patients were suspected to have a focal intimal flap proximal to the M1 occlusion site. The first patient became irritable and complained of severe headache during passage of the microcatheter through the occluded lesion and died due to massive bleeding caused by rupture of the false lumen. The six patients who underwent contact aspiration thrombectomy showed an intimal flap and double lumen in the occluded M1 site after initial EVT. Of these patients, five
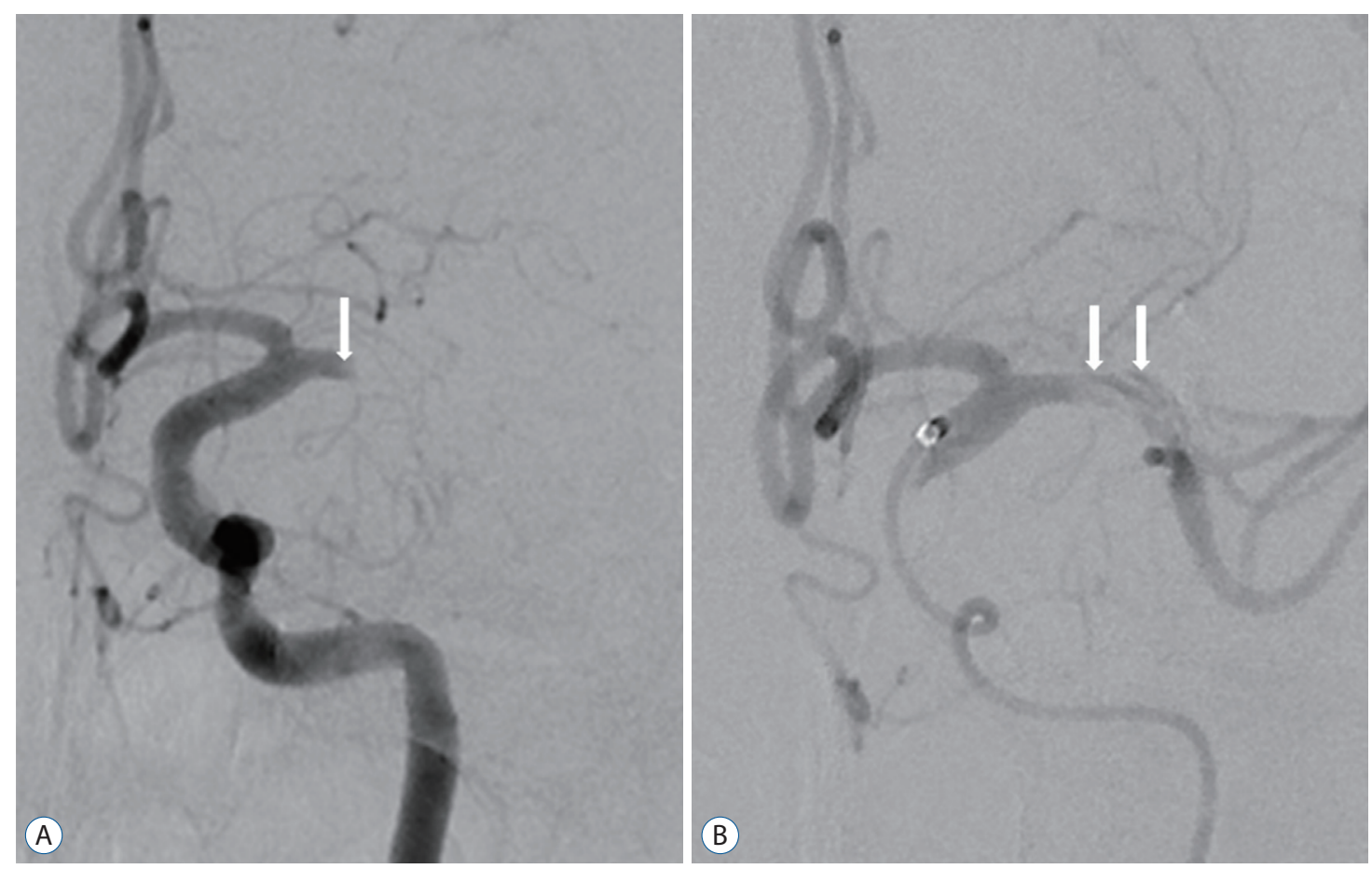

Fig. 3. A 45-year-old man with complete occlusion of left M1. A : Left internal carotid angiogram showing complete obstruction of the left middle cerebral artery with focal intimal flap (arrow). B : Left internal carotid angiogram after initial contrast aspiration thrombectomy using a Penumbra catheter showing a double lumen and intimal flap (arrows). The patient was discharged without neurological complications. 
(83.8\%) had a good clinical outcome (modified Rankin Scale score $\leq 2$ ). A stent was placed in the MCA of one patient due to progressive symptoms.

\section{DISCUSSION}

Isolated MCA dissection is a rare clinical finding, but it is an important cause of stroke. It may present as an isolated dissection related to ischemia or local symptoms or as a dissecting aneurysm with rupture leading to SAH or ICH, both of which are associated with high morbidity and mortality ${ }^{9}$.

Invasive and noninvasive methods have been utilized to detect MCA dissection. Cerebral angiography remains the gold standard for identifying cervicocephalic arterial dissections ${ }^{1,10}$. The typical angiographic findings of MCA dissection are similar to those of dissection of the extracranial arteries : string sign, irregular stenosis, pseudoaneurysm, and eventually total occlusion. However, a definitive diagnosis of MCA dissection can be difficult as pathognomonic features of dissection are detected in fewer than $10 \%$ of dissected arteries ${ }^{3,5}$. Further, it can be difficult to distinguish between a cardioembolism and an occlusion caused by MCA dissection when determining cause of stroke based on initial angiographic findings. In our study, only $2.4 \%$ of patients had acute MCA occlusion related to MCA dissection. For each of these patients, a subtle intimal flap proximal to the occlusion site was retrospectively detected.

Passage of a microcatheter and microguidewire through an MCA occlusion related to MCA dissection should be undertaken with utmost care due to the unclear entry site of a true and false lumen. If a microcatheter is advanced into a false lumen (as in case 1; Fig. 2), wide extension of the false lumen can occur and rupture of an intracranial artery can result. The patient in case 1 became irritable and had a severe headache during catheter passage through the obstructive lesion. Severe headache is the most common symptom for patients with intracranial dissection. We believed her severe headache was caused by expansion of the false lumen, as often occurs in patients with an underlying MCA dissection or iatrogenic MCA dissection.

Appropriate management of MCA dissection remains controversial. Treatment strategies include use of antiplatelet agents, anticoagulation, thrombolysis, surgery, EVT, and con- servative therapy. Treatment for ischemic symptoms due to stenosis caused by dissection or SAH due to a dissecting aneurysm should be selected based on symptom severity and the location of the dissection. Surgical and conservative therapies can lead to good outcomes in patients with dissecting aneurysms of the anterior cerebral artery or MCA. Medical treatments using intravenous recombinant tissue plasminogen activator or antiplatelet therapy offer good clinical outcomes without clinical complications ${ }^{4}$. We should have considered an endovascular approach using Penumbra aspiration thrombectomy as first-line treatment because of complete occlusion of the M1 segment coupled with acute neurologic symptoms. Therefore, we suggest patients with suspected MCA dissection should undergo contrast aspiration thrombectomy without passage of a microcatheter. Also, additional cerebral angiography should be done to confirm the extent of false lumen and double lumen and to check collateral status for evaluation of clinical outcome.

\section{CONCLUSION}

We demonstrated that complete occlusion related to MCA dissection is difficult to diagnose with certainty due to pathognomonic features of the dissection that may not be revealed in angiography. We suggest that a small intimal flap proximal to a completely occluded MCA site should raise the possibility of MCA dissection. The first option for EVT with patients with MCA dissection and complete occlusion should be contact aspiration thrombectomy without passage of a microcatheter.

\section{CONFLICTS OF INTEREST}

No potential conflict of interest relevant to this article was reported.

\section{INFORMED CONSENT}

Informed consent was obtained from all individual participants included in this study. 


\section{AUTHOR CONTRIBUTIONS}

\author{
Conceptualization : HSK \\ Data curation : KHP, JSP \\ Formal analysis : KHP, JSP \\ Methodology : HSK \\ Project administration : HSK \\ Visualization : KHP, JSP \\ Writing - original draft : KHP, JSP \\ Writing - review \& editing: HSK
}

\section{ORCID}

\begin{abstract}
Kang-Hoon Park https://orcid.org/0000-0003-2822-8907
Hyo Sung Kwak https://orcid.org/0000-0002-7228-8117

Jung-Soo Park https://orcid.org/0000-0002-4949-203X
\end{abstract}

\section{References}

1. Ahn SS, Kim BM, Suh SH, Kim DJ, Kim DI, Shin YS, et al. : Spontaneous symptomatic intracranial vertebrobasilar dissection: initial and follow-up imaging findings. Radiology 264 : 196-202, 2012

2. Asaithambi G, Saravanapavan P, Rastogi V, Khan S, Bidari S, Khanna AY, et al. : Isolated middle cerebral artery dissection: a systematic review. Int J Emerg Med 7 : 44, 2014

3. Beletsky V, Norris JW : Spontaneous dissection of the carotid and verte- bral arteries. N Engl J Med 345 : 467, 2001

4. Doijiri R, Yokota C, Suzuki R, Toyoda K, Minematsu K : Intravenous recombinant tissue plasminogen activator thrombolysis in acute ischemic stroke due to middle cerebral artery dissection. J Stroke Cerebrovasc Dis 21 : 915.E7-915.E9, 2012

5. Houser OW, Mokri B, Sundt TM Jr, Baker HL Jr, Reese DF : Spontaneous cervical cephalic arterial dissection and its residuum: angiographic spectrum. AJNR Am J Neuroradiol 5 : 27-34, 1984

6. Kurino M, Yoshioka $S$, Ushio $Y$ : Spontaneous dissecting aneurysms of anterior and middle cerebral artery associated with brain infarction: a case report and review of the literature. Surg Neurol 57 : 428-436; discussion 436-438, 2002

7. Kwak HS, Hwang SB, Chung GH, Jeong SK : High-resolution magnetic resonance imaging of symptomatic middle cerebral artery dissection. J Stroke Cerebrovasc Dis 23 : 550-553, 2014

8. Lee HO, Kwak HS, Chung GH, Hwang SB : Diagnostic usefulness of high resolution cross sectional MRI in symptomatic middle cerabral arterial dissection. J Korean Neurosurg Soc 49 : 370-372, 2011

9. Lee JS, Bang OY, Lee PH, Kim BM, Yong SW : Two cases of spontaneous middle cerebral arterial dissection causing ischemic stroke. J Neurol Sci 250 : 162-166, 2006

10. Ohkuma H, Suzuki S, Kikkawa T, Shimamura N : Neuroradiologic and clinical features of arterial dissection of the anterior cerebral artery. AJNR Am J Neuroradiol 24 : 691-699, 2003

11. Ohkuma H, Suzuki S, Shimamura N, Nakano $T$ : Dissecting aneurysms of the middle cerebral artery: neuroradiological and clinical features. Neuroradiology 45 : 143-148, 2003

12. Vertinsky AT, Schwartz NE, Fischbein NJ, Rosenberg J, Albers GW, Zaharchuk $\mathrm{G}$ : Comparison of multidetector $\mathrm{CT}$ angiography and MR imaging of cervical artery dissection. AJNR Am J Neuroradiol 29 : 1753-1760, 2008 